\title{
Comparison of the Composition of Three Mahonia Plants Based on GC-MS Analysis
}

\author{
Haitao Guo ${ }^{1.2}$, Xiaoming Xue ${ }^{1,2^{*}}$, Chenghui Nan ${ }^{1.2}$, Xi Liu ${ }^{1}$, Zehui Wang ${ }^{3}$, and Bin Dao ${ }^{3}$ \\ ${ }^{1}$ Nanjing Forest Police College, Nanjing, 210023, China; \\ ${ }^{2}$ Key Laboratory of National Forestry and Grassland Administration on Wildlife Evidence Technology, Nanjing,210023, China \\ ${ }^{3}$ Yunnan Forestry Police Bureau, kunming, 650224, China
}

\begin{abstract}
Plant material evidence inspection is Mahonia bealei (Fort.) Carr. , Mahonia fortunei (Lindl.) and Mahonia bodinieri Gagnep. are three common medicinal plants commonly found in Mahonia. In this study, gas chromatography-mass spectrometry (GC-MS) was used to compare the chemical constituents of stems and leaves of these three plants. The results showed that 6 of the volatile oils of the three plant species contained the same chemical composition, which was neophytadiene, palmitic acid, n-dodecane, octacosane, erucamide, and vitamin $\mathrm{E}$ oil, but the percentage content was difference. The main components of the volatile oils of the three plant stems were different. Erucamide was only detected in Mahonia fortunei (Lindl.) Fedde, oleic acid amide was only detected in Mahonia bealei (Fort.) Carr. , and oxidized cyclooctene ,and four compounds such as triphenylphosphine oxide was detected in Mahonia bodinieri Gagnep. ..
\end{abstract}

\section{Introduction}

Mahonia plants belong to Bebreridaeeae shrubs or small trees. There are about 100 species in the world, mainly distributed in East Asia, Southeast Asia, North America, Central America and Western South America. There are about 35 species in China ${ }^{[1]}$. The genus is mainly planted with roots and stems. It is called thorn berberine, thorn jasper and gongcao wood. In some areas, it is also used as a leaf for its leaves. Therefore, roots, stems and leaves of Mahonia plants can be used as medicine. It is used as a folk medicine and traditional medicine in various distribution areas in China. It has the functions of purging fire and detoxifying, nourishing yin and tonifying the lungs, clearing away heat and dampness, and also nourishing the liver and kidneys ${ }^{[2]}$. It is mainly distributed in Sichuan, Guizhou, Yunnan and southeastern China. This study analyzed and compared the composition of Mahonia fortunei (Lindl.) Fedde, Mahonia bealei (Fort.) Carr. and Mahonia bodinieri Gagnep.

There are many reports on the chemical composition of Mahonia plants. For the three chemical studies of Mahonia fortunei, Liu Yuxiang et al. ${ }^{[3]}$ used steam distillation to extract volatile oil from Mahonia bodinieri Gagnep. The chemical constituents were identified by gas chromatography-mass spectrometry (GC-MS) and the chemical constituents were identified by gas chromatography. More than 50 components were separated from the volatile oil of Mahonia bodinieri Gagnep. 36 compounds were identified, accounting for more than $90 \%$ of the total. Mahonia bodinieri Gagnep.
The main components of the volatile oil of the leaves are: palmitic acid (54.49\%), and the higher content is linoleic acid $(5.98 \%)$, a -farnesene $(5.51 \%)$, methyl linolenic acid $(3.45 \%)$. , methyl palmitate $(3.36 \%)$ and the like.

Dong Lei et $\mathrm{al}^{\left[{ }^{[4]}\right.}$ used steam distillation to extract volatile oil from the stem of Mahonia bealei (Fort.) Carr., and determined the relative percentage of each component in volatile oil by normalization method and combined it with gas chromatography-mass spectrometry (The chemical composition of the structure was identified by GC-MS. A total of 96 components were detected and 18 of them were identified, accounting for more than $80 \%$ of the total volatile oil. Dong Lei et al. ${ }^{[5]}$ also used steam distillation to extract volatile oil from Mahonia bealei (Fort.) Carr. leaves, and detected a total of 155 components, and identified 28 compounds, accounting for more than $50.6 \%$ of the total volatile oil. Fan $\mathrm{Libo}^{[6]}$ and others extracted ethyl acetate extracts from the stems and leaves of Mahonia fortunei ( Lindl. ) Fedde with ethyl acetate. The extracts were concentrated and purified by repeated silica gel column and gel column chromatography to obtain 5 compounds. By physical and chemical properties and TLC, 1H NMR, 13C NMR, MS analysis, the structure of the compound was identified as berberine (I), jatrorrhizine (II), 5methoxy zephyr product D (III) (Note: for the first time Isolated from this genus), luteolin (IV) and $\beta$-sitosterol (V). This extraction method is cumbersome and complicated to operate, but the obtained extract has high purity.

Liu Yuxiang ${ }^{[3]}$, Dong Lei ${ }^{[4,5]}$ and others use steam distillation to extract volatile oil, which is only suitable

* Corresponding author: xuexm@nfpc.edu.cn 
for good volatility, can be distilled without steam, does not react with water, and is insoluble or Extraction of water-insoluble ingredients. The method has the advantages of simple equipment, simple operation, high safety, low cost, high oil yield and no pollution to the environment. However, this method also has some shortcomings. During the operation, Mahonia fortunei plants are susceptible to heat, easy to coke, or change the chemical composition $^{[7]}$, so that the extracted volatile oil components are inaccurate, resulting in gas chromatography-mass spectrometry analysis is an error.

\section{Materials and methods}

\subsection{Sample collection and processing}

In March 2017, Mahonia bealei (Fort.) Carr. was collected at Nanjing Zhongshan Botanical Garden; Mahonia fortunei was collected at Nanjing Forest Police College; Mahonia bodinieri Gagnep. was collected in south eastern Guizhou. The three kinds of Mahonia fortunei collected were naturally air-dried, and then crushed into powders by a grinder to obtain $15 \mathrm{~g}$ of each powder, which was placed in a sample bag for use.

\subsection{Instruments and reagents}

Laboratory equipment: METTLER TTOLEdo (model: MT5), gas chromatography-mass spectrometer (Agilent Technologies 7890A GC system, 5975C inter XL MSD Agilent, USA) with autosampler, KQ-250B ultrasonic cleaning (Kunshan Ultrasonic Instrument Co., Ltd.), TG16-WS desktop high-speed centrifuge (Shanghai Luxiangyi Centrifuge Instrument Co., Ltd.), grinding machine.

\subsection{Gas Chromatography-Mass Spectrometry Analysis}

The method of extracting the components of volatile oil by double liquid phase: weigh $3 \mathrm{~g}$ of stem and leaf powder of three Mahonia fortunei, respectively, and place them in a $10 \mathrm{ml}$ centrifuge tube, and add $9 \mathrm{ml}$ of $1: 1$ n-hexane/anhydrous ethanol to mix ultrasonic aid. The mixture was extracted for 30 minutes, repeated twice, and left to stand at room temperature for 30 minutes, and then centrifuged for 10 minutes by means of a centrifuge (rotation speed: $40 \times 100 \mathrm{r} / \mathrm{min}$ ), and the supernatant was extracted and used.

\subsubsection{Gas chromatographic conditions}

The column is HP-5MS GC column with a column length of $30 \mathrm{~m}$, an inner diameter of $0.25 \mathrm{~mm}$ and a membrane thickness of $0.25 \mu \mathrm{m}$. The carrier gas is highpurity nitrogen, and the carrier gas saves $20 \mathrm{ml} / \mathrm{min}$; the split ratio is 20:1; the syringe size is $10 \mathrm{ul}$. Sample volume $1 \mathrm{ul}$; pressure $8.2317 \mathrm{psi}(1 \mathrm{MPa}=145 \mathrm{psi})$; flow rate $1 \mathrm{ml} / \mathrm{min}$; after running $1 \mathrm{~min}$; programmed temperature: initial temperature $60^{\circ} \mathrm{C}, 20^{\circ} \mathrm{C} / \mathrm{min}$ to $320^{\circ} \mathrm{C}$, for $5 \mathrm{~min}$; solvent delay $3 \mathrm{~min}$

\subsubsection{Mass spectrometry conditions}

Ion mode $\mathrm{EI}$; ion source temperature $230^{\circ} \mathrm{C}$, maximum $250{ }^{\circ} \mathrm{C}$; quadrupole temperature $150^{\circ} \mathrm{C}$, maximum $200^{\circ} \mathrm{C}$; transmission line temperature $230^{\circ} \mathrm{C}$; solvent delay $3 \mathrm{~min}$.

\section{Results}

The total ionograms of the six samples were obtained by gas chromatography-mass spectrometry. The peaks of the total ion currents were scanned by mass spectrometry to obtain the mass spectra of the components, and then the mass spectra of the six samples were identified by mass spectrometry database. The percentage of each compound in the volatile oil of 6 samples was calculated according to the peak area normalization method.

\subsection{Chemical composition analysis of leaf volatile oil}

Three kinds of Mahonia leaf volatile oil were analyzed by GC-MS and identified as chemical components, as shown in Figure 1 and Table 1. The same ingredients of the three are neophytadiene, palmitic acid, n-octadecane, octacosane, erucamide, and vitamin $\mathrm{E}$ oil. The total content of the six components is Mahonia bealei (high to low). Fort.) Carr. (85.370\%), Mahonia bodinieri Gagnep. (65.207\%), Mahonia fortunei (54.506\%). Among them, Mahonia fortunei and Mahonia bodinieri Gagnep. contain phytosterols of $8.486 \%$ and $7.199 \%$, respectively, but Mahonia bealei (Fort.) Carr. does not have this ingredient. Mahonia fortunei and Mahonia bealei (Fort.) Carr. contain linolenic acid, 3.101\% and 3.079\%, respectively, but Mahonia bodinieri Gagnep. does not have this ingredient.

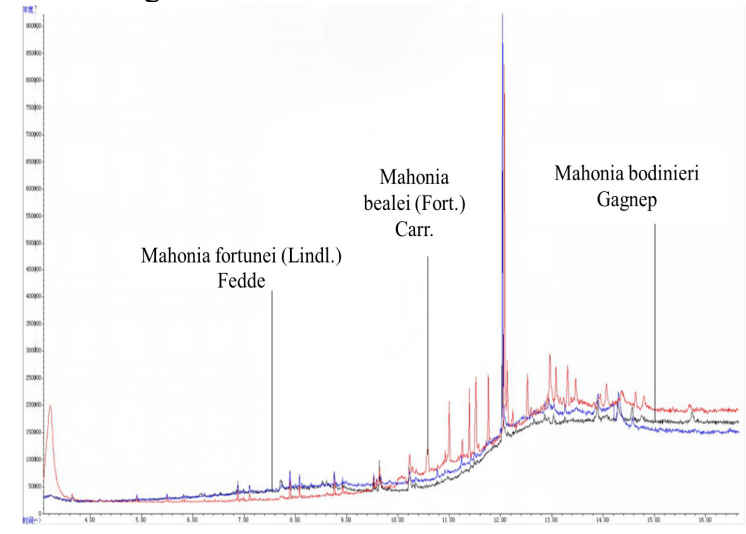

Fig. 1. Total ion flow diagram of leaf volatile oil of Mahonia fortunei, Mahonia bealei (Fort.) Carr. And Mahonia bodinieri Gagnep. 
Table 1. Volatile oil composition of Mahonia fortunei, Mahonia bealei (Fort.) Carr. And Mahonia bodinieri Gagnep

\begin{tabular}{|c|c|c|c|c|}
\hline Component name & $\begin{array}{l}\text { structural } \\
\text { formula }\end{array}$ & $\begin{array}{c}\text { Mahonia } \\
\text { fortunei content } \\
(\%)\end{array}$ & $\begin{array}{l}\text { Mahonia bealei } \\
\text { (Fort.) Carr. content } \\
(\%)\end{array}$ & $\begin{array}{c}\text { Mahonia bodinieri } \\
\text { Gagnep. content } \\
(\%)\end{array}$ \\
\hline 3-Tetradecanone & $\mathrm{C}_{14} \mathrm{H}_{28} \mathrm{O}$ & 7.226 & 0 & 0 \\
\hline $\begin{array}{l}\text { Neophytadiene } \\
3,7,11,15-\end{array}$ & $\mathrm{C}_{20} \mathrm{H}_{38}$ & 8.859 & 9.771 & 9.857 \\
\hline $\begin{array}{l}\text { Tetramethylhexadec-2-en- } \\
\text { 1-yl acetate }\end{array}$ & $\mathrm{C}_{22} \mathrm{H}_{42} \mathrm{O}_{2}$ & 3.261 & 0 & 0 \\
\hline n-Hexadecanoic acid & $\mathrm{C}_{16} \mathrm{H}_{32} \mathrm{O}_{2}$ & 3.695 & 4.697 & 1.498 \\
\hline Octacosane & $\mathrm{C}_{28} \mathrm{H}_{58}$ & 1.234 & 17.327 & 8.154 \\
\hline Nonacosane & $\mathrm{C}_{29} \mathrm{H}_{60}$ & 5.106 & 16.254 & 7.598 \\
\hline 13-Docosenamide, & $\mathrm{C}_{22} \mathrm{H}_{43} \mathrm{NO}$ & 13.071 & 28.608 & 24.408 \\
\hline Vitamin E & $\mathrm{C}_{29} \mathrm{H}_{50} \mathrm{O}_{2}$ & 22.541 & 8.713 & 13.692 \\
\hline $\begin{array}{l}\text { 9,12-Octadecadienoic acid } \\
(\mathrm{Z}, \mathrm{Z})\end{array}$ & $\mathrm{C}_{18} \mathrm{H}_{30} \mathrm{O}_{2}$ & 3.079 & 3.101 & 0 \\
\hline .beta.-Sitosterol & $\mathrm{C}_{29} \mathrm{H}_{50} \mathrm{O}$ & 8.486 & 0 & 7.199 \\
\hline Tetracosane & $\mathrm{C}_{24} \mathrm{H}_{50}$ & 8.709 & 0 & 0 \\
\hline $\begin{array}{l}\text { 1,2,5-Oxadiazol-3-amine,4- } \\
\text { (4-meth oxyphenoxy) }\end{array}$ & $\mathrm{C}_{9} \mathrm{H}_{9} \mathrm{~N}_{3} \mathrm{OS}$ & 3.411 & 0 & 0 \\
\hline 13-Hexacosyne & $\mathrm{C}_{26} \mathrm{H}_{50}$ & 3.763 & 0 & 0 \\
\hline 17-Pentatriacontene & $\mathrm{C}_{35} \mathrm{H}_{70}$ & 6.669 & 0 & 0 \\
\hline $\begin{array}{l}\text { 1,4-Benzenediol,2,5-bis } \\
\text { (1,1-dimethylethyl) - 2,5- }\end{array}$ & $\mathrm{C}_{14} \mathrm{H}_{22} \mathrm{O}_{2}$ & 0 & 5.539 & 0 \\
\hline $\begin{array}{c}\text { 9,12,15-Octadecatrien-1-ol, } \\
(\mathrm{Z}, \mathrm{Z}, \mathrm{Z}) \text { - }\end{array}$ & $\mathrm{C}_{18} \mathrm{H}_{32} \mathrm{O}$ & 0 & 5.990 & 0 \\
\hline $\begin{array}{l}\text { Bicyclo[4.1.0]heptane, 3- } \\
\text { methyl }\end{array}$ & $\mathrm{C}_{6} \mathrm{H}_{10} \mathrm{O}_{2}$ & 0 & 0 & 2.121 \\
\hline 1-Hexadecyne 1 & $\mathrm{C}_{16} \mathrm{H}_{30}$ & 0 & 0 & 30.145 \\
\hline Octacosanal & $\mathrm{C}_{28} \mathrm{H}_{56} \mathrm{O}$ & 0 & 0 & 4.281 \\
\hline $\begin{array}{l}\text { 1,1'-Biphenyl,3,3',4,4',5,5'- } \\
\text { hexamethoxy- }\end{array}$ & $\mathrm{C}_{18} \mathrm{H}_{22} \mathrm{O}_{6}$ & 0 & 0 & 5.304 \\
\hline $\begin{array}{c}\text { Benzenepropanenitrile,.beta } \\
\text {.-phenyl- } 3,3\end{array}$ & $\mathrm{C}_{15} \mathrm{H}_{13} \mathrm{~N}$ & 0 & 0 & 4.872 \\
\hline $\begin{array}{c}\text { Eicosane } \\
\text { Berbine, 13,13a-didehydro- }\end{array}$ & $\mathrm{C}_{20} \mathrm{H}_{42}$ & 0 & 0 & 3.431 \\
\hline $\begin{array}{c}\text { 9,10-dim ethoxy-2,3- } \\
\text { (methylenedioxy) }\end{array}$ & $\mathrm{C}_{20} \mathrm{H}_{19} \mathrm{NO}_{4}$ & 0 & 0 & 7.442 \\
\hline
\end{tabular}

The specific chemical composition of Mahonia fortunei is from tetradecane $(8.709 \%)$ and 3tetradecanone $(7.226 \%)$, and the specific chemical composition of Mahonia bealei (Fort.) Carr. is 2,5. - Ditert-butyl hydroquinone (5.539\%); Mahonia bodinieri Gagnep. The specific chemical composition percentages from high to low are 1-hexadecane $(30.145 \%), 3,3-$ diphenylpropionitrile $\quad(4.872 \%)$, dihydroberberine (7.442\%), analytical pure n-icosane (3.431\%).

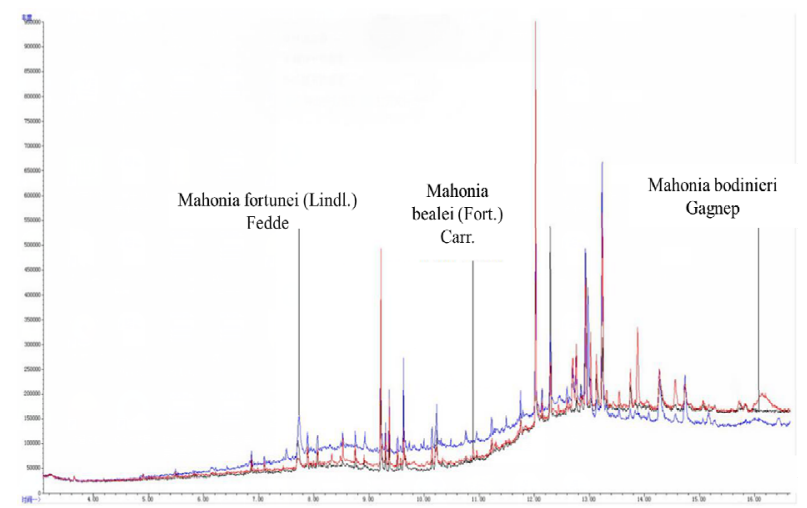

Fig. 2. Total ion flow diagram of stem volatile oil of Mahonia fortunei, Mahonia bealei (Fort.) Carr. And Mahonia bodinieri Gagnep 
Table 2. Composition of essential oil from stem of Mahonia fortunei, Mahonia bealei (Fort.) Carr. And Mahonia bodinieri Gagnep

\begin{tabular}{c|cccc}
\hline Component name & structural formula & $\begin{array}{c}\text { Mahonia fortunei } \\
\text { content }(\%)\end{array}$ & $\begin{array}{c}\text { Mahonia bealei (Fort.) } \\
\text { Carr. content (\%) }\end{array}$ & $\begin{array}{c}\text { Mahonia bodinieri } \\
\text { Gagnep. content }(\%)\end{array}$ \\
\hline 9-Octadecenamide, $(\mathrm{Z})$ & $\mathrm{C}_{18} \mathrm{H}_{35} \mathrm{NO}$ & 0 & 100 & 0 \\
13-Docosenamide, $(\mathrm{Z})$ & $\mathrm{C}_{22} \mathrm{H}_{43} \mathrm{NO}$ & 100 & 0 & 23.399 \\
$\begin{array}{c}\text { 9-Oxabicyclo[6.1.0]nonane } \\
\text { Cyclodecasiloxane, } \\
\text { eicosamethyl }\end{array}$ & $\mathrm{C}_{8} \mathrm{H}_{14} \mathrm{O} \mathrm{H}_{60} \mathrm{O}_{10} \mathrm{Si}_{10}$ & 0 & 0 & 27.756 \\
$\begin{array}{c}\text { Triphenylphosphine oxide } \\
\text { 2,5-Dihydroxybenzoic acid, } \\
\text { 3TMS derivative }\end{array}$ & $\mathrm{C}_{7} \mathrm{H}_{3} \mathrm{D}_{3} \mathrm{O}_{4}$ & 0 & 0 & 4.589 \\
$\quad \mathrm{C}_{18} \mathrm{H}_{15} \mathrm{OP}$ & 0 & 0 & 9.87 \\
$\begin{array}{c}\text { (E) -2- } \\
\text { bromobutyloxychalcone }\end{array}$ & $\mathrm{C}_{16} \mathrm{H}_{50} \mathrm{O}_{7} \mathrm{Si}_{8}$ & 0 & 0 & 11.191 \\
$\begin{array}{c}\text { Pyridine-3-carboxamide, } \\
\text { oxime,N- (2- }\end{array}$ & $\mathrm{C}_{15} \mathrm{H}_{13} \mathrm{~N}$ & 0 & 0 & 10.385 \\
trifluoromethylphenyl)-3, 3 & & 0 & 0 & 12.810 \\
\hline
\end{tabular}

\subsection{Chemical analysis of volatile oil from stem}

Three kinds of Mahonia fortunei stem volatile oil were analyzed by GC-MS and identified as chemical components, as shown in Figure 2 and Table 2. Mahonia fortunei contains only erucamide; Mahonia bealei (Fort.) Carr. contains only oleic acid amide; Mahonia bodinieri Gagnep. contains more chemical components, the percentages from high to low are erucamide $(23.399 \%)$ Cyclooctene oxide $(27.756 \%)$, triphenylphosphine oxide (11.191\%), 3,3-diphenylpropionitrile $(12.810 \%)$ accounting for $75.156 \%$ of the total content.

\section{Discussion}

\subsection{Comparison of chemical components between stem and leaf parts}

In this study, gas chromatography-mass spectrometry was used to analyze the volatile oil samples of stems and leaves of Mahonia fortunei, Mahonia bealei (Fort.) Carr. and Mahonia bodinieri Gagnep. 10 chemical components, such as 3-tetradecanone, neophytadiene, erucic acid amide, palmitic acid, phytosterols, etc., were isolated from the leaves of Mahonia fortunei, accounting for $82.896 \%$ of the total volatile oil. Only erucamide was isolated and identified, and its stem and leaves contained erucamide, which were $100 \%$ and $13.071 \%$, respectively. Separation and identification of 9 chemical components from the leaves of Mahonia bealei (Fort.) Carr., such as di-tert-butyl hydroquinone, linolenic acid, erucamide, ndodecane, octacosane, palmitic acid, etc. The main component accounts for $94.01 \%$ of the total volatile oil. Only the oleic acid amide is isolated and identified from the stem, and its stem and leaves contain different components. 11 chemical components were isolated from the leaves of Mahonia bealei (Fort.) Carr., such as dihydroberberine, analytical pure n-icosane, 3,3diphenylpropionitrile, 1-hexadecane, plant Alcohol, neophytadiene, erucamide, vitamin E oil and other main components accounted for $88.294 \%$ of the total volatile oil. Four chemical components were isolated from the stem and identified as erucamide, cyclooctene oxide and triphenyl oxide. Phosphine and 3,3-diphenylpropionitrile accounted for $75.156 \%$ of the total volatile oil, and the stem and leaves contained erucamide and 3,3diphenylpropionitrile, respectively, $34.59 \%$ and $29.28 \%$.

\subsection{Comparison of chemical composition of three Mahonia fortunei}

In this study, gas chromatography-mass spectrometry was used to analyze the volatile oil samples of Mahonia fortunei, Mahonia bealei (Fort.) Carr. and Mahonia bodinieri Gagnep. stems and leaves, and 14 components were isolated from the volatile oil of Mahonia fortunei stem and leaf. 10 chemical constituents; 10 components were isolated from the volatile oil of Mahonia bealei (Fort.) Carr. stems and leaves, and 9 chemical components were identified; 20 components were isolated from the volatile oil of Mahonia bodinieri Gagnep. stems and leaves, identified 15 chemical components. The leaves of three species of Mahonia fortunei contain six chemical components, including neophytadiene, palmitic acid, n-octadecane, octacosane, erucamide, and vitamin E oil, which fully exemplify these three Mahonia fortunei in chemistry. The same nature of the ingredients. But the unique 4-butanone of Mahonia fortunei, the unique 2,5-di-tert-butyl hydroquinone, oleic acid amide, Mahonia bodinieri Gagnep, unique dihydroberberine, Mahonia bealei (Fort.) Carr. Analysis of pure eicosane, 1-hexadecane, 3,3diphenylpropionitrile, oxidized cyclooctene and other chemical components, also visually reflects their compositional differences.

Fan Libo et al ${ }^{[6]}$ extracted the ethanol extracts from the stems and leaves of Mahonia fortunei with ethyl acetate. The extracts were concentrated and separated and purified by repeated silica gel column and gel column chromatography. Five compounds were identified. This study did not detect; Dong Lei et al [4] extracted the volatile oil from the stems and leaves of Mahonia bealei (Fort.) Carr. by steam distillation, and analyzed by gas chromatography-mass spectrometry. The 32 chemical components were not detected in this study; Liu Yuxiang et al ${ }^{[3]}$ used steam distillation to extract volatile oil from the stems and leaves of Mahonia 
bodinieri Gagnep. After gas chromatography-mass spectrometry analysis, it was identified. 36 chemical components were obtained, of which palmitic acid was also detected in this study, but the content was different. The results of this study were $4.697 \%$, and Dong Lei et $\mathrm{al}^{[4]}$ showed $54.49 \%$, and the other components were not detected.

This difference should be caused by the extraction method. In this study, the extraction method of the two liquid phase with n-hexane and absolute ethanol as the extractant is used. The difference of the extraction reagent will directly affect the detection result. The separation and identification of this experiment are identified. No ingredients have been reported except for palmitic acid.

\section{Conclusion}

In this study, the chemical constituents of the stem and leaf samples of Mahonia fortunei, Mahonia bealei (Fort.) Carr. and Mahonia bodinieri Gagnep. were analyzed and summarized. The results show that the method of extracting volatile oil components in two liquid phase is feasible and effective.

The three-liquid phase extraction method of three species of Mahonia fortunei volatile oil from stems and leaves, using anhydrous ethanol and n-hexane as extractants, can be reflected by gas chromatographymass spectrometry (GC-MS) analysis. The same chemical composition is 6 kinds of neophytadiene, palmitic acid, n-octadecane, octacosane, erucamide and vitamin $\mathrm{E}$ oil. The difference in chemical composition is reflected in both quantity and type. In terms of quantity, Mahonia fortunei has identified 10 chemical components, Mahonia bealei (Fort.) Carr. has identified 9 chemical components, and Mahonia bodinieri Gagnep. has identified 15 chemical components. Chemical composition; species differences are reflected in the unique chemical composition of these three Mahonia fortunei, Mahonia fortunei contains 3-tetradecanone, Mahonia bealei (Fort.) Carr. contains 2,5-di-tert-butyl hydroquinone, oil Acid amide, Mahonia bodinieri Gagnep. contains dihydroberberine, analytical pure nicosane, 1-hexadecane, 3,3-diphenylpropionitrile, oxidized cyclooctene.

\section{Acknowledgement}

This work is financially supported by the Fundamental Research Funds for he Central Universities (Grant Nos. LGZD201704). and Natural science of Jiangsu Province (Grant Nos. BK20181338).

\section{References}

1. X.Z. Wang, L.S, 3(2010)

2. Y.F. Zhang, Journal of Zhejiang Public Security College, 78(2003)

3. Y.F. Zhang, Chinese Journal of Forensic Medicine, 18 (2003)
4. H.Z. Qin, Southeast University Press, 2007

5. Chinese Flora Editorial Board of the Chinese Academy of Sciences. Chinese Flora (Vol. XX). Beijing: Science Press, 217(2001)

6. Y.Z. Zhang, H.J. Yang, J.J. Yang Junjie. Journal of Xinyang Agriculture and Forestry College,23(2013)

7. Y.X. Liu, B.M. Liu, X. M. Dong, Journal of Guangxi Academy of Sciences, 26(2010)

8. L. Dong, X. H .Yang, Y. Wang, et al. Journal of Changchun University of Traditional Chinese Medicine,22(2006).

9. L. Dong, F. H. Yan, X. H .Yang, et al. Specialty Research, 30(2008)

10. L.Fan, X. H .Zhang, X. G. Liu, et al. Chinese Journal of Veterinary Drug, 45(2011)

11. J.X Li, K.X. Wang, C. P. Li. Shizhen Guo Guo Guo Yao, 19(2008)

12. Y.X. Chen, H. F. Shi, X.J. Song, et al. Journal of Sichuan Agricultural University, 34(2016) 